\title{
Evaluation and Comparison of IMRT versus 3DCRT for Post-Mastectomy Chest Wall Irradiation
}

\author{
Pritanjali Singh ${ }^{1}$, Vinita Kumari ${ }^{2}$, Pawan Kumar ${ }^{3}$ \\ ${ }^{1}$ Associate Professor, Department of Radiotherapy, All India Institute of Medical Sciences, Patna, Bihar, India, ${ }^{2}$ Senior Resident, Department of Radiotherapy, All \\ India Institute of Medical Sciences, Patna, Bihar, India, ${ }^{3}$ Medical Physicist, Department of Radiotherapy, All India Institute of Medical Sciences, Patna, Bihar, India.
}

\section{Abstract}

Background: Breast cancer is the most common cancer in females worldwide. Hence, we planned the present study to evaluate and compare IMRT and 3DCRT for post-mastectomy chest wall irradiation. Subjects and Methods: A total of 30 subjects were included in the present study. Adjuvant radiotherapy was planned in all the subjects to the chest wall. A Lead marker was used for marking the scar sites, drain sites and breast borders. Non-contrast computed tomography (CT) simulation and treatment was carried out in all the patients. Generation of Planning Target Volume (PTV) was done followed by contouring of organs at risk (OAR). 15 subjects were treated with three-dimensional conformal radiation therapy (3DCRT) and 15 subjects were with intensity-modulated radiation therapy (IMRT) for post-mastectomy radiotherapy (PMRT). Calculation of conformity index (CI) was done as per criteria previously described in the literature. Calculation of mean dose volume of ipsilateral lung and heart was done. All the results were summarized and analysed by SPSS software. Results: Significant results were obtained while comparing the Dmean (Gy) for heart and lungs in between the 3DCRT group and the IMRT group. Conclusion: In comparison to 3DCRT, among post-mastectomy left sided breast cancer patients, IMRT offered significant reduction in the dose volume of the ipsilateral lung and heart.

Keywords: Intensity Modulated Radiotherapy, Three-Dimensional Planned Conformal Radiotherapy.

Corresponding Author: Dr. Vinita Kumari, Senior Resident, Department of Radiotherapy, All India Institute of Medical Sciences, Patna, Bihar, India.

Received: May 2019

Accepted: May 2019

\section{Introduction}

Breast cancer is the most common cancer in females worldwide. Large prospective trials and a meta-analysis have shown that adjuvant radiotherapy of the chest wall improves local control and survival in node positive breast cancer patients after mastectomy. Randomized, retrospective and population based studies have shown that the radiotherapy of the chest wall is associated with a significantly increased risk of developing ipsilateral second lung cancer, and in patients treated on the left side with a significantly increased risk of cardiac morbidity and mortality. ${ }^{[1-3]}$

There is a good body of literature showing that inversed planned intensity modulated radiotherapy (IMRT) potentially leads to a more favourite dose distribution compared to three-dimensional planned conformal radiotherapy (3D-CRT) for the radiotherapy of the whole breast after breast conserving surgery. ${ }^{[4-6]}$ Under the light of above mentioned data, we planned the present study to evaluate and compare IMRT and 3DCRT for postmastectomy chest wall irradiation.

\section{Subjects and Methods}

The present study was conducted in the Department of
Radiotherapy, All India Institute of Medical Sciences, Patna, Bihar (India) and it included evaluation and comparison of IMRT versus 3DCRT for post-mastectomy chest wall irradiation. Ethical approval was obtained from institutional ethical committee and written consent was obtained from all the subjects after explaining in detail the entire research protocol.

Inclusion criteria for the present study included:

- Subjects more than 20 years of age,

- Subjects with previous untreated post-MRM female breast cancer patients,

- Subjects with histopathologic confirmed diagnosis of infiltrating ductal carcinoma,

- Subjects with absence of evidence of distant metastasis or second malignancy

After meeting the inclusion criteria, a total of 30 subjects were included in the present study. Adjuvant radiotherapy was planned in all the subjects to the chest wall. A Lead marker was used for marking the scar sites, drain sites and breast borders. Non-contrast computed tomography (CT) simulation and treatment was carried out in all the patients. As per radiation Therapy Oncology Group (RTOG) breast cancer atlas guidelines, clinical target volume (CTV) was contoured. ${ }^{[5]}$

Generation of Planning Target Volume (PTV) was done 
followed by contouring of organs at risk (OAR). 15 subjects were treated with three-dimensional conformal radiation therapy (3DCRT) and 15 subjects were with intensitymodulated radiation therapy (IMRT) for post-mastectomy radiotherapy (PMRT). Calculation of conformity index (CI) was done as per criteria previously described in the literature. ${ }^{[7]}$

Calculation of mean dose volume of ipsilateral lung and heart was done. All the results were summarized and analysed by SPSS software.

\section{Results}

In the present study, a total of 30 subjects were analyzed. Mean age of the subjects of the 3DCRT group and IMRT group was 53.8 years and 55.1 years respectively. Mean BMI of the subjects of the 3DCRT group and IMRT group was $25.3 \mathrm{Kg} / \mathrm{m} 2$ and $26.1 \mathrm{Kg} / \mathrm{m} 2$ years respectively. Dmean (Gy) for lungs for the 3DCRT group and the IMRT group was 14.5 and 12.5 Gy respectively. Dmean (Gy) for heart for the 3DCRT group and the IMRT group was 6.11 and 3.14 Gy respectively. Significant results were obtained while comparing the Dmean (Gy) for heart and lungs in between the 3DCRT group and the IMRT group.

Table 1: Demographic data
\begin{tabular}{|l|l|l|}
\hline Parameter & 3DCRT group & IMRT group \\
\hline Mean age (years) & 53.8 & 55.1 \\
\hline Mean BMI (Kg/m2) & 25.3 & 26.1 \\
\hline Menopausal status - Pre & 5 & 6 \\
\hline Menopausal status - Post & 10 & 9 \\
\hline
\end{tabular}

Table 2: Dosimetric comparison

\begin{tabular}{|l|l|l|l|}
\hline Parameter & $\begin{array}{l}\text { 3DCRT } \\
\text { group }\end{array}$ & $\begin{array}{l}\text { IMRT } \\
\text { group }\end{array}$ & p- value \\
\hline CTV chest (mL) & 320.2 & 322.5 & 0.15 \\
\hline PTV volume (mL) & 615.2 & 617.2 & 0.41 \\
\hline $\begin{array}{l}\text { Dmean (Gy) for } \\
\text { lungs }\end{array}$ & 14.5 & 12.5 & 0.01 (Significant) \\
\hline Dmean (Gy) for heart & 6.11 & 3.14 & 0.01 (Significant) \\
\hline Conformity index & 1.35 & 1.11 & 0.00 (Significant) \\
\hline
\end{tabular}

\section{Discussion}

Although the utilization of breast conserving surgery (BCS) for early-stage disease has increased rapidly in last decade in the whole world, modified radical mastectomy (MRM) remains the most-accepted surgical modality in operable breast cancer. Three randomized clinical trials have shown that a disease-free and overall survival advantage is conferred by the addition of chest wall and regional lymph node irradiation in women with positive axillary lymph nodes after MRM. ${ }^{[7-9]}$

In the present study, a total of 30 subjects were analyzed. Mean age of the subjects of the 3DCRT group and IMRT group was 53.8 years and 55.1 years respectively. Mean BMI of the subjects of the 3DCRT group and IMRT group was $25.3 \mathrm{Kg} / \mathrm{m} 2$ and $26.1 \mathrm{Kg} / \mathrm{m} 2$ years respectively. Huang XB et al evaluated the potential dosimetric benefits and optimal indications of intensity-modulated radiotherapy
(IMRT) for the intact breast. Ten patients with stage Tis2N0M0 breast cancer, who received breast-conserving surgery, were selected for this study. The maximum dose $(\mathrm{D}(\max ))$ of coronary artery decreased from $5057.1 \mathrm{cGy}$ to 4832.9 cGy, and the mean dose (D(mean)) of heart decreased from 629.8 cGy to 450.7 cGy; the Dmean of liver decreased from $283.9 \mathrm{cGy}$ to $172.0 \mathrm{cGy}$ for right sided patients; the Dmean and percentage of volume receiving more than 20 Gy (V20) of ipsilateral lung decreased from 925.2 cGy to $765.9 \mathrm{cGy}$, and from $16.0 \%$ to $15.3 \%$, respectively; the Dmean and V20 of different central lung distance (CLD) subgroups decreased by $14.7 \%$ and $20.9 \%$, $7.0 \%$ and $12.9 \%$, respectively; the Dmean of contralateral breast decreased from 75.4 cGy to 20.3 cGy; the Dmean of contralateral lung decreased from 30.9 cGy to $16.1 \mathrm{cGy}$. Forward planning IMRT based on a standard tangential beam arrangement significantly improves the dose homogeneity throughout the target volume of intact breast, and reduces the dose to OARs, especially in patients with large breast volumes or exceeded CLD, who might be proposed as candidates of IMRT for intact breast. ${ }^{[9]}$

In our study, Dmean (Gy) for lungs for the 3DCRT group and the IMRT group was 14.5 and 12.5 Gy respectively. Dmean (Gy) for heart for the 3DCRT group and the IMRT group was 6.11 and 3.14 Gy respectively. Significant results were obtained while comparing the Dmean (Gy) for heart and lungs in between the 3DCRT group and the IMRT group. Rudat $\mathrm{V}$ et al compared the surface dose of 7-field IMRT (7 F-IMRT), tangential beam IMRT (TB-IMRT), and tangential beam 3D-CRT (3D-CRT) of breast cancer patients receiving adjuvant radiotherapy by means of in vivo GafChromic film dosimetry. Breast cancer patients receiving adjuvant radiotherapy of the whole breast or the chest wall were eligible for the study. Study patients were treated with a treatment plan using two different radiotherapy techniques (first patient series, 3D-CRT followed by TB-IMRT; second patient series, TB-IMRT followed by 7 F-IMRT). The surface dose was evaluated on three consecutive treatment fractions per radiotherapy technique using in vivo GafChromic film dosimetry. In our study, the paired t-test was used to assess the difference of in vivo GafChromic film readings or calculated plan parameters of the compared pairs of radiation techniques for statistical significance. Forty-five unselected breast cancer patients were analysed in this study. 7 F-IMRT significantly reduced the surface dose compared to TB-IMRT. Differences were greatest in the central and lateral breast or chest wall region and amounted to a dose reduction of $11.8 \%$ to $-18.8 \%$. No significant difference of the surface dose was observed between TB-IMRT and 3D-CRT. A corresponding observation was obtained for the calculated skin dose derived from dose-volume histograms. In adjuvant breast cancer radiotherapy, 7 F-IMRT offers a significantly reduced surface dose compared to TB-IMRT or 3D-CRT. ${ }^{[10]}$ 


\section{Conclusion}

Under the light of above obtained data, the authors conclude that in comparison to 3DCRT, among post-mastectomy left sided breast cancer patients, IMRT offered significant reduction in the dose volume of the ipsilateral lung and heart.

\section{References}

1. El Saghir NS, Khalil MK, Eid T, El Kinge AR, Charafeddine M, Geara F, Seoud M, Shamseddine AI. Trends in epidemiology and management of breast cancer in developing Arab countries: a literature and registry analysis. Int J Surg. 2007;5(4):225-33.

2. Habermann EB, Abbott A, Parsons HM, Virnig BA, Al-Refaie WB, Tuttle TM. Are mastectomy rates really increasing in the United States? J Clin Oncol. 2010;28(21):3437-41.

3. Overgaard M, Nielsen HM, Overgaard J. Is the benefit of postmastectomy irradiation limited to patients with four or more positive nodes, as recommended in international consensus reports? A subgroup analysis of the DBCG 82 b\&c randomized trials. Radiother Oncol. 2007;82(3):247-53.

4. Berrington de Gonzalez A, Curtis RE, Gilbert E, Berg CD, Smith SA, Stovall M, Ron E. Second solid cancers after radiotherapy for breast cancer in SEER cancer registries. Br J Cancer. 2010;102(1):220-26.

5. Moorthy S, Sakr H, Hasan S, Samuel J, Al-Janahi S, Murthy N.
Dosimetric study of SIB-IMRT versus SIB-3DCRT for breast cancer with breath-hold gated technique. Int $\mathrm{J}$ Cancer Ther Oncol. 2013;1:010110.

6. RTOG Foundation . Philadelphia, PA: RTOG Foundation; c2018. Breast cancer atlas [Internet] [cited 2018 Mar 1]. Available from: https://www.rtog.org/CoreLab/ContouringAtlases/BreastCancerAtlas. aspx. [Google Scholar]

7. International Commission on Radiation Units \& Measurements . Bethesda, MD: International Commission on Radiation Units \& Measurements; c2018. Prescribing, recording, and reporting intensity-modulated photon-beam therapy (IMRT) (ICRU Report 83) [Internet] [cited 2018 Mar 1]. Available from:https://icru.org/testing/reports/prescribing-recordingandreporting-intensity-modulated-photon-beam-therapy-imrticrureport-83.

8. Hong L, Hunt M, Chui C, et al. Intensity-modulated tangential beam irradiation of the intact breast. Int $\mathrm{J}$ Radiat Oncol Biol Phys. 1999;44:1155-64.

9. Huang XB1, Jiang GL, Chen JY, Chen LF, Hu WG. Dosimetric evaluation of intensity-modulated tangential beam versus conventional tangential irradiation for breast cancer. Ai Zheng. 2006 Jul;25(7):855-60.

10. Rudat V1, Nour A, Alaradi AA, Mohamed A, Altuwaijri S. In vivo surface dose measurement using GafChromic film dosimetry in breast cancer radiotherapy: comparison of 7-field IMRT, tangential IMRT and tangential 3D-CRT. Radiat Oncol. 2014 Jul 15;9:156. doi: 10.1186/1748-717X-9-156.

Copyright: (c) the author(s), 2019. It is an open-access article distributed under the terms of the Creative Commons Attribution License (CC BY 4.0), which permits authors to retain ownership of the copyright for their content, and allow anyone to download, reuse, reprint, modify, distribute and/or copy the content as long as the original authors and source are cited.

How to cite this article: Singh P, Kumari V, Kumar P. Evaluation and Comparison of IMRT versus 3DCRT for Post-Mastectomy Chest Wall Irradiation. Asian J. Med. Res. 2019;8(2):MC01-MC03.

DOI: dx.doi.org/10.21276/ajmr.2019.8.2.MC1

Source of Support: Nil, Conflict of Interest: None declared. 